\title{
A generalized Clark-Ocone formula
}

\author{
Margarida de FARIA ${ }^{1}$, Maria João OLIVEIRA ${ }^{2}$ and Ludwig STREIT ${ }^{1,3}$ \\ ${ }^{1}$ CCM, Universidade da Madeira, $P 9000$ Funchal \\ ${ }^{2}$ Univ. Aberta/Grupo de Física Matemática, Av. Prof. Gama Pinto, 2, P 1649-003 Lisboa \\ ${ }^{3}$ BiBoS, Universität Bielefeld, D 33501 Bielefeld
}

Received for ROSE November 24, 1998

\begin{abstract}
We extend the Clark-Ocone formula to a suitable class of generalized Brownian functionals. As an example we derive a representation of Donsker's delta function as (limit of) a stochastic integral.
\end{abstract}

\section{INTRODUCTION}

For suitable functionals $\varphi$ of Brownian motion, expressed in terms of Itô integrals

$$
\varphi=\mathbf{E}(\varphi)+\int m(\tau) \mathrm{d} B(\tau)
$$

the Clark-Ocone formula [5], [14] provides us with an explicit formula for the integrand $m(\cdot)$, given $\varphi$. It has become clear that such an expression should be useful in the determination of hedging portfolios, see e.g., [1], [3], [15]. Another application is in the context of determining the quadratic variation process of Brownian martingales, see e.g., [7] for a recent example.

On the other hand it was pointed out in [2] that the conditions on $\varphi$ are restrictive. It seems desirable to extend the validity of (1) and of the Clark-Ocone formula. A possible setting is that of generalized functionals of white noise as described, e.g., in [8]-[13], [16]. In particular the generalized function space elaborated in [16], or the larger one of [8], retain the probabilistic properties that are required for such a generalization. In [3] one finds an announcement of results in terms of the Potthoff-Timpel [4], [16] space (in the meantime elaborated in [1]), for related results in the space $D^{\prime}$ of Malliavin calculus see [17]. Here we use the space of [8], which we present in the following section together with extensions of the Skorohod and Itô integrals, the gradient and some further auxiliary notions. Section 3 has our generalization of the Clark-Ocone formula, in Section 4 we translate the result into the language, often useful in practical calculations, of the $S$-transform. A case in point is Donsker's $\delta$-function for which we elaborate the generalized Clark-Ocone formula in Section 5.

\section{REGULAR GENERALIZED FUNCTIONS OF WHITE NOISE}

\subsection{Regular generalized functions}

We will recall the definition and some properties of the space $\mathcal{G}^{-1}$ of regular generalized functions of White Noise [8], [9]. 
Within the Hilbert space $L_{d}^{2}(\mathbb{R}) \equiv L^{2}\left(\mathbb{R}, \mathbb{R}^{d}\right), d \in \mathrm{N}$, of vector valued square integrable functions we consider the space $S_{d}(\mathbb{R})$ of vector valued Schwartz test functions. The topology on $S_{d}(\mathbb{R})$ may be given in terms of a system of increasing Hilbertian norms

$$
|\vec{\xi}|_{p}^{2}=\sum_{i=1}^{d}\left|\xi_{i}\right|_{p}^{2}, \vec{\xi}=\left(\xi_{1}, \ldots, \xi_{d}\right) \in S_{d}(\mathbb{R}), \xi_{i} \in S(\mathbb{R}), i=1, \ldots, d, p \in \mathrm{N}_{0}
$$

The basic nuclear triple is thus

$$
S_{d}(\mathbb{R}) \subset L_{d}^{2}(\mathbb{R}) \subset S_{d}^{\prime}(\mathbb{R}) .
$$

On $S_{d}^{\prime}(\mathbb{R})$ we fix the canonical Gaussian measure $\mu_{d}$ which is determined by the characteristic function

$$
C(\vec{\xi}) \equiv \exp \left(-\frac{1}{2} \sum_{\mathrm{i}=1}^{\mathrm{d}} \int_{\mathbb{R}} \xi_{\mathrm{i}}^{2}(\mathrm{t}) \mathrm{dt}\right), \vec{\xi} \in \mathrm{S}_{\mathrm{d}}(\mathbb{R}) .
$$

The space $L^{2}\left(S_{d}^{\prime}(\mathbb{R}), \mu_{d}\right)$ will be briefly denoted by $\left(L^{2}\right)$.

We will denote by $\vec{n}$ the $d$-tuple $\left(n_{1}, \ldots, n_{d}\right), n_{i} \in \mathrm{N}_{0}$, and write

$$
\begin{aligned}
n & =\sum_{i=1}^{d} n_{i}, \\
\vec{n} ! & =\prod_{i=1}^{d} n_{i} ! .
\end{aligned}
$$

The norm and the inner product in $L^{2}\left(\mathbb{R}^{n}\right)$ will be denote by $|\cdot|_{n}$ and $(\cdot, \cdot)_{n}$, respectively.

Considering square integrable white noise functionals $\varphi$ for which the chaos expansion

$$
\begin{aligned}
\varphi(\vec{\omega}) & =\sum_{\vec{n}}<: \vec{\omega}^{\otimes \vec{n}}:, \varphi_{\vec{n}}> \\
& \equiv \sum_{\vec{n}} \int_{\mathbb{R}^{n}} d^{n} t \varphi_{\vec{n}}\left(t_{1}^{1}, \ldots, t_{n}^{d}\right) \prod_{i=1}^{d}: \omega_{i}^{\otimes n_{i}}:\left(t_{1}^{i}, \ldots, t_{n_{\mathrm{i}}}^{i}\right)
\end{aligned}
$$

converges rapidly, i.e.,

$$
\|\varphi\|_{q}^{2} \equiv \sum_{\vec{n}}(\vec{n} !)^{2} 2^{q n}\left|\varphi_{\vec{n}}\right|_{n}^{2}<\infty,
$$

we define the Hilbert space $G_{q}^{1}$ as

$$
G_{q}^{1}=\left\{\varphi \in\left(L^{2}\right):\|\varphi\|_{q}^{2}<\infty\right\} .
$$

The space of test functions $\mathcal{G}^{1}$ is defined as the projective limit of the spaces $G_{q}^{1}, q \in \mathrm{N}_{0}$,

$$
\mathcal{G}^{1}=p r-\lim _{q} G_{q}^{1}
$$

Let $G_{-q}^{-1}$ be the dual with respect to $\left(L^{2}\right)$ of $G_{q}^{1}$ and $\mathcal{G}^{-1}$ the dual space of $\mathcal{G}^{1}$ with respect to $\left(L^{2}\right)$. The corresponding bilinear dual pairing $\ll \cdot, \cdot \gg$ is connected to the sesquilinear inner product on $\left(L^{2}\right)$ by

$$
\ll F, \varphi \gg=(\bar{F}, \varphi)_{\left(L^{2}\right)} \text {, if } F \in\left(L^{2}\right) \text {. }
$$


(We shall use the same notation $\ll \cdot, \cdot \gg$ for dual pairings in more general settings such as, e.g., for $L^{2}(\mathbb{R}) \otimes \mathcal{G}^{ \pm 1}$ ). Since the constant function 1 is in $\mathcal{G}^{1}$ we may extend the definition of the expectation $\mathbf{E}(\cdot)$ from integrable functions to distributions $\Phi \in \mathcal{G}^{-1}$ :

$$
\mathbf{E}(\Phi)=\ll \Phi, 1 \gg \text {. }
$$

From general duality theory it follows that

$$
\mathcal{G}^{-1}=\bigcup_{q \geq 0} G_{-q}^{-1}
$$

therefore, every distribution is of finite order, i.e., for every $\Phi \in \mathcal{G}^{-1}$ there exists $q \in \mathrm{N}_{0}$ such that $\Phi \in G_{-q}^{-1}$. It turns out from the definition that the Hilbert space $G_{-q}^{-1}$ can be described as follows:

$$
G_{-q}^{-1}=\left\{\Phi(\vec{\omega})=\sum_{\vec{n}}<: \vec{\omega}^{\otimes \vec{n}}:, \Phi_{\vec{n}}>,\|\Phi\|_{-q}^{2} \equiv \sum_{\vec{n}} 2^{-q n}\left|\Phi_{\vec{n}}\right|_{n}^{2}<\infty\right\} .
$$

Given $\vec{\xi} \in S_{d}(\mathbb{R})$, let us consider the Wick exponential

$$
\begin{aligned}
: \exp \langle\vec{\omega}, \vec{\xi}\rangle: & \equiv \exp \left(\langle\vec{\omega}, \vec{\xi}\rangle-\frac{1}{2} \sum_{i=1}^{d} \int \xi_{i}^{2}(t) d t\right) \\
& =\sum_{\vec{n}} \frac{1}{\vec{n} !}\left\langle: \vec{\omega}^{\otimes \vec{n}}:, \vec{\xi}^{\otimes \vec{n}}\right\rangle, \vec{\omega} \in S_{d}^{\prime}(\mathbb{R}) .
\end{aligned}
$$

Since the sum

$$
\sum_{\vec{n}}(\vec{n} !)^{2} 2^{q n}\left|\frac{1}{\vec{n} !} \vec{\xi}^{\otimes \vec{n}}\right|_{L_{d}^{2}\left(\mathbb{R}^{n}\right)}^{2}=\sum_{\vec{n}} 2^{q n}|\vec{\xi}|_{L_{d}^{2}(\mathbb{R})}^{2 n}
$$

converges if and only if $2^{q}|\bar{\xi}|_{L_{d}^{2}(\mathbb{R})}^{2}<1$, the Wick exponentials are not test functions in $\mathcal{G}^{1}$, but they are in those $G_{q}^{1}$ for which $2^{q}|\vec{\xi}|_{L_{d}^{2}(\mathbb{R})}^{2}<1$. Thus it is still possible to define an $S$-transform in the space $\mathcal{G}^{-1}$ because every distribution is of finite order. Given $\Phi \in \mathcal{G}^{-1}$, there exists $q \in \mathrm{N}_{0}$ such that $\Phi \in G_{-q}^{-1}$. For all $\vec{\xi} \in S_{d}(\mathbb{R})$ with $2^{q}|\vec{\xi}|_{L_{d}^{2}(\mathbb{R})}^{2}<1$ we define the $S$-transform of $\Phi$ as

$$
S \Phi(\vec{\xi}) \equiv \ll \Phi,: \exp \langle\cdot \vec{\xi}\rangle: \gg=\sum_{\vec{n}}\left(\Phi_{\vec{n}}, \vec{\xi}^{\otimes \vec{n}}\right)_{n} .
$$

This definition extends to complex vectors $\vec{\eta} \in S_{d, c}(\mathbb{R})$ such that $2^{q}|\vec{\eta}|_{L_{d}^{2}(\mathbb{R})}^{2}<1$,

$$
S \Phi(\vec{\eta}) \equiv \ll \Phi,: \exp <\cdot, \vec{\eta}>: \gg=\sum_{\vec{n}}\left(\Phi_{\vec{n}}, \vec{\eta}^{\otimes \vec{n}}\right)_{n} .
$$

Therefore, for $\Phi \in G_{-q}^{-1}$, (2) defines the $S$-transform for every $\vec{\eta}$ from the open neighborhood of zero, $U_{q} \equiv\left\{\vec{\eta} \in S_{d, c}(\mathbb{R}): 2^{q}|\vec{\eta}|_{L_{d}^{2}}^{2}(\mathbb{R})<1\right\}, q \in \mathbf{N}_{0}$. 


\subsection{Generalization of the gradient operator}

We begin with the observation that the Hida derivative $\partial_{t}$ fails to be pointwise defined on the spaces $G_{ \pm q}^{ \pm 1}$. However we still may consider the gradient $\left(\partial^{i} \cdot{ } \cdot\right)_{1 \leq i \leq d}$ as an operator

$$
\left(\partial^{i} \cdot\right)_{1 \leq i \leq d}: G_{ \pm q}^{ \pm 1} \rightarrow \mathbb{R}^{d} \otimes L^{2}(\mathbb{R}) \otimes G_{ \pm p}^{ \pm 1} .
$$

Given $\psi$ a test function from $\mathcal{G}^{1}$ with kernel functions $\psi_{\vec{n}}, n \in \mathrm{N}_{0}$, we define the operator gradient of $\psi, \nabla \psi \equiv\left(\partial^{i} . \psi\right)_{1 \leq i \leq d}$, where, for each $1 \leq i \leq d, \partial^{i} . \psi$ is the functional from $L^{2}(\mathbb{R}) \otimes G_{q}^{1}, q \in \mathrm{N}_{0}$, characterized by the sequence

$$
\begin{aligned}
\psi_{\vec{n}}^{i}(t, s) \equiv\left(n_{i}+1\right) \psi \overrightarrow{n+\delta_{i}}\left(s_{1}^{1}, \ldots, s_{n_{1}}^{1} ; \ldots ; s_{1}^{i}, \ldots, s_{n_{i}}^{i}, t ; \ldots ; s_{1}^{d}, \ldots, s_{n_{d}}^{d}\right) & \in L^{2}(\mathbb{R}) \otimes L^{2}\left(\mathbb{R}^{n}\right), \\
& \vec{n}=\left(n_{1}, \ldots, n_{d}\right) .
\end{aligned}
$$

In fact,

$$
\begin{aligned}
\int_{\mathbb{R}} \mathrm{d} t \sum_{\vec{n}}(\vec{n} !)^{2} 2^{q n}\left|\psi_{\vec{n}}^{i}(t, \cdot)\right|_{n}^{2} & =\sum_{\vec{n}}\left(\left(\overrightarrow{n+\delta_{i}}\right) !\right)^{2} 2^{q n}\left|\psi \overrightarrow{n+\delta_{i}}\right|_{n+1}^{2} \\
& =2^{-q} \sum_{\vec{n}, n \geq 1}(\vec{n} !)^{2} 2^{q n}\left|\psi_{\vec{n}}\right|_{n}^{2},
\end{aligned}
$$

which proves that $\partial^{i} . \psi \in L^{2}(\mathbb{R}) \otimes G_{q}^{1}$ for every non negative integer number $q$ and, moreover, the continuity of the linear operators $\partial_{.}^{i}: G_{q}^{1} \rightarrow L^{2}(\mathbb{R}) \otimes G_{q}^{1}$ :

$$
\left\|\partial_{.}^{i} \psi\right\|_{L^{2}(\mathbb{R}) \otimes G_{q}^{1}}^{2} \leq 2^{-q}\|\psi\|_{G_{q}^{1}}^{2}, \psi \in \mathcal{G}^{1},
$$

for every $q \in \mathrm{N}_{0}$. Hence,

$$
\sum_{i=1}^{d}\left\|\partial_{.}^{i} \psi\right\|_{L^{2}(\mathbb{R}) \otimes G_{q}^{1}}^{2} \leq d \cdot 2^{-q}\|\psi\|_{G_{q}^{1}}^{2}, \psi \in \mathcal{G}^{1},
$$

which proves that the linear operator $\nabla: G_{q}^{1} \rightarrow \mathbb{R}^{d} \otimes L^{2}(\mathbb{R}) \otimes G_{q}^{1}$ is continuous.

We extend the operator gradient from test functions on $\mathcal{G}^{1}$ (introduced above) to distributions on $\mathcal{G}^{-1}$.

Given a regular generalized function $\Phi$ from $\mathcal{G}^{-1}, \Phi \in G_{-q}^{-1}$ for some $q \in \mathrm{N}_{0}$, characterized by the sequence $\left(\Phi_{\vec{n}}\right), n \in \mathbb{N}_{0}, \Phi_{\vec{n}} \in L^{2}\left(\mathbb{R}^{n}\right)$, consider the functional characterized by the sequence

$$
\begin{aligned}
& \Phi_{\bar{n}}^{i}(t, s) \equiv\left(n_{i}+1\right) \Phi \underset{n+\delta_{i}}{\longrightarrow}\left(s_{1}^{1}, \ldots, s_{n_{1}}^{1} ; \ldots ; s_{1}^{i}, \ldots, s_{n_{\mathrm{i}}}^{i}, t ; \ldots ; s_{1}^{d}, \ldots, s_{n_{\mathrm{d}}}^{d}\right) \in L^{2}(\mathbb{R}) \otimes L^{2}\left(\mathbb{R}^{n}\right), \\
& n \in \mathbb{N}_{0} .
\end{aligned}
$$

Using the inequality $2^{-k} k^{2}<2$ for $k=(p-q) n \geq 0$, we have

$$
\begin{aligned}
\int_{\mathbb{R}} \mathrm{d} t \sum_{\vec{n}} 2^{-p n}\left(n_{i}+1\right)^{2} & \left|\Phi_{\overrightarrow{n+\delta_{i}}}(\cdot, t, \cdot)\right|_{n}^{2}=\sum_{\vec{n}} 2^{-p n}\left(n_{i}+1\right)^{2}\left|\Phi_{\overrightarrow{n+\delta_{i}}}\right|_{n+1}^{2} \\
& =2^{p} \sum_{\vec{n}} 2^{-q(n+1)} 2^{-(p-q)(n+1)}\left(n_{i}+1\right)^{2}\left|\Phi_{\overrightarrow{n+\delta_{i}}}\right|_{n+1}^{2} \\
& \leq \frac{2^{p+1}}{(p-q)^{2}} \sum_{\vec{n}} 2^{-q(n+1)}\left|\Phi_{\overrightarrow{n+\delta_{i}}}\right|_{n+1} \\
& =\frac{2^{p+1}}{(p-q)^{2}} \sum_{\vec{n}, n \geq 1} 2^{-q n}\left|\Phi_{\vec{n}}\right|_{n}^{2},
\end{aligned}
$$


where the above sum is convergent because $\Phi \in G_{-q}^{-1}$. Hence, the sequence $\left(\Phi_{\vec{n}}^{i}\right)$, $n \in \mathbb{N}_{0}$, defines a functional from $L^{2}(\mathbb{R}) \otimes G_{-p}^{-1}$. Keeping the terminology and the notation introduced above, we will denote this functional by $\partial^{i} \Phi$ and the operator $\left(\partial^{i} \Phi\right)_{1 \leq i \leq d}$ will be called the gradient of $\Phi$, denoted by $\nabla \Phi$. Using this notation, it follows from (3) that

$$
\|\nabla \Phi\|_{\mathbb{R}^{d} \otimes L^{2}(\mathbb{R}) \otimes G_{-p}^{-1}}^{2} \leq d \frac{2^{p+1}}{(p-q)^{2}}\|\Phi\|_{G_{-q}^{-1}}^{2},
$$

for every pair $p>q \geq 0$, which proves that the gradient is a bounded linear operator from $G_{-q}^{-1}$ into $\mathbb{R}^{d} \otimes L^{2}(\mathbb{R}) \otimes G_{-p}^{-1}$ if $p>q$.

\subsection{An extension of the Skorohod and Itô integrals}

In [10] the Skorohod integral was discussed in a white noise setting. An extension to certain generalized white noise integrands can be found in [6].

Considering an element $\Phi$ from $L^{2}(\mathbb{R}) \otimes G_{-q}^{-1}$, for some $q \in N_{0}$, characterized by the sequence $\Phi_{\vec{n}}(\cdot ; \cdot) \in L^{2}(\mathbb{R}) \otimes L^{2}\left(\mathbb{R}^{n}\right), n \in \mathrm{N}_{0}$, let us consider the functional characterized by the sequence

$$
\begin{aligned}
\Psi_{0}^{i} & \equiv 0 \\
\Psi_{\vec{n}}^{i} & \equiv \tilde{\Phi}_{\overrightarrow{n-\delta_{i}}} \in L^{2}\left(\mathbb{R}^{n}\right), \vec{n}=\left(n_{1}, \ldots, n_{d}\right), n \in N,
\end{aligned}
$$

where $\tilde{\Phi}_{\overrightarrow{n-\delta_{i}}}$ denotes the symmetrization of $\Phi_{\overrightarrow{n-\delta_{i}}}$ in the variables $t, s_{1}^{i}, \ldots, s_{n_{i}-1}^{i}$. Since, for each $1 \leq i \leq d$,

$$
\begin{aligned}
\sum_{\vec{n}, n \geq 1} 2^{-q n}\left|\tilde{\Phi}_{\overline{n-\delta_{i}}}\right|_{n}^{2} & \leq\left.\left.\sum_{\vec{n}, n \geq 1} 2^{-q n}\right|_{\Phi_{n-\delta_{i}}}\right|_{L^{2}(\mathbb{R}) \otimes L^{2}\left(\mathbb{R}^{n-1}\right)} ^{2} \\
& =2^{-q} \int_{\mathbb{R}} \mathrm{d} t \sum_{\bar{n}} 2^{-q n}\left|\Phi_{\bar{n}}(t ; \cdot)\right|_{n}^{2} \\
& =2^{-q}\|\Phi\|_{L^{2}(R) \otimes \mathrm{G}_{-q}^{-1}}^{2},
\end{aligned}
$$

the sequence $\left(\Psi_{\tilde{n}}^{i}\right), n \in \mathbb{N}_{0}$, defines a distribution from $G_{-q}^{-1}$. We denote it by $I_{i}(\Phi)$. For every test function $\psi$ from $\mathcal{G}^{1}$ with kernel functions $\left(\psi_{\vec{n}}\right), n \in \mathbf{N}_{0}$, we have for each $1 \leq i \leq d$,

$$
\begin{aligned}
& \ll I_{i}(\Phi), \psi \gg=\sum_{\vec{n}, n \geq 1} \vec{n} !\left(\tilde{\Phi}_{\overrightarrow{n-\delta_{i}}}, \psi_{\vec{n}}\right)_{n} \\
& =\sum_{\vec{n}, n \geq 1} \vec{n} !\left(\Phi_{\overrightarrow{n-\delta_{i}}}, \psi_{\vec{n}}\right)_{L^{2}(\mathbb{R}) \otimes L^{2}\left(\mathbb{R}^{n-1}\right)}^{n} \\
& =\int_{\mathbb{R}} \mathrm{d} t \sum_{\vec{n}, n \geq 1}\left(\overrightarrow{n-\delta_{i}}\right) !\left(\Phi \underset{\overrightarrow{n-\delta_{i}}}{\longrightarrow}(t ; \cdot), n_{i} \psi_{\vec{n}}(\cdot, t, \cdot)\right)_{n-1} \\
& =\int_{\mathbb{R}} \mathrm{d} t \ll \Phi(t ; \cdot), \partial_{t}^{i} \psi \gg=\ll \Phi, \partial^{i} \psi \gg,
\end{aligned}
$$

and $I_{i}(\Phi)$ is the unique functional from $\mathcal{G}^{-1}$ for which the above equality holds for every test function $\psi \in \mathcal{G}^{1}$; if $I^{\prime}(\Phi)$ is another functional in such conditions, it turns out that $\ll I_{i}(\Phi)-I^{\prime}(\Phi), \cdot \gg$ is identically equal to zero on $\mathcal{G}^{1}$. 
We may now formulate the

Definition. Given $\Phi$ an element from $\mathbb{R}^{d} \otimes L^{2}(\mathbb{R}) \otimes G_{-q}^{-1}$, for some $q \in \mathrm{N}_{0}$, we call generalized Skorohod integral of $\Phi$ the distribution on $\mathcal{G}^{-1}, I(\Phi)$, defined by the sum

$$
I(\Phi) \equiv \sum_{i=1}^{d} I_{i}\left(\Phi_{i}\right)
$$

where, for each $i=1, \ldots, d, I_{i}\left(\Phi_{i}\right)$ is the unique regular generalized function from $\mathcal{G}^{-1}$ for which the following equality

$$
\left\langle\left\langle I_{i}\left(\Phi_{i}\right), \psi\right\rangle\right\rangle=\left\langle\left\langle\Phi_{i}, \partial^{i} \psi\right\rangle\right\rangle
$$

holds for every test function $\psi$ from $\mathcal{G}^{1}$.

This definition generalizes the notion of Skorohod integral. In fact, in the particular situation $\Phi \in \mathbb{R}^{d} \otimes L^{2}(\mathbb{R}) \otimes D$,

$$
D \equiv\left\{F \in\left(L^{2}\right): F(\omega)=\sum_{\vec{n}}<: \omega^{\otimes \vec{n}}:, F_{\vec{n}}>, \sum_{\vec{n}} \vec{n} ! n\left|F_{\vec{n}}\right|_{n}^{2}<\infty\right\},
$$

the generalized Skorohod integral $I(\Phi)$ coincides with the Skorohod integral. In view of the relation between the Skorohod and Itô integral we may add the following remark.

Remark 1. For $t \in \mathbb{R}$, let $\mathcal{F}_{t}$ denote the $\sigma$-algebra generated by the random variables $\{B(s), s \leq t\}$, where $\left(B_{t}\right)_{t \in \mathbb{R}}$ is a $d$-dimensional Brownian motion. If $F \in$ $\mathbb{R}^{d} \otimes L^{2}(\mathbb{R}) \otimes\left(L^{2}\right)$ and it is adapted to the filtration $\left(\mathcal{F}_{t}\right)_{t \in \mathbb{R}}$, then the generalized Skorohod integral $I(F)$ is equal to the Itô integral of $F$. Without the first condition we speak of a generalized Itô integral.

Before ending this subsection, we return to the sequence of inequalities (4) which imply

$$
\left\|I_{i}\left(\Phi_{i}\right)\right\|_{G_{-q}^{-1}}^{2} \leq 2^{-q}\left\|\Phi_{i}\right\|_{L^{2}(\mathbb{R}) \otimes G_{-q}^{-1}}^{2}
$$

i.e., $I$ is a bounded linear operator from $\mathbb{R}^{d} \otimes L^{2}(\mathbb{R}) \otimes \mathrm{G}_{-\mathrm{q}}^{-1}$ into $\mathrm{G}_{-\mathrm{q}}^{-1}, q \in \mathrm{N}_{0}$.

\subsection{Some notations and definitions}

For the next sections it is useful to introduce some notations and recall some definitions.

We shall denote by $\Theta_{t}$ the Heaviside function

$$
\Theta_{t}(s) \equiv\left\{\begin{array}{l}
1 \text { if } s \leq t \\
0 \text { if } s>t
\end{array}\right.
$$

and also the linear operator given by

$$
\Theta_{t}: f(\cdot) \rightarrow \Theta_{t}(\cdot) f(\cdot) .
$$

The functional derivatives $\frac{\delta}{\delta f(t)}$, for suitable $\mathrm{G}$, are defined as

$$
\lim _{\varepsilon \rightarrow 0} \frac{G\left(f+\varepsilon f_{0}\right)-G(f)}{\varepsilon}=\int f_{0}(t) \frac{\delta G(f)}{\delta f(t)} \mathrm{d} t .
$$


In particular for cylinder functions $G(f)=g\left(\int h(t) f(t) \mathrm{d} t\right)$ where $g$ is a differentiable function, then

$$
\frac{\delta G(f)}{\delta f(\tau)}=g^{\prime}\left(\int h(t) f(t) \mathrm{d} t\right) h(\tau) .
$$

For what follows it is also helpful to define the notion of second quantization of $\Theta_{t}$, $t \in \mathbb{R}$, defined on the space $\mathcal{G}^{-\infty}$. Recall:

For bounded linear operators $A$ on $L^{2}(\mathbb{R})$ the linear map which transforms each sequence $\left(\varphi_{\vec{n}}\right), n \in \mathrm{N}_{0}, \varphi_{\vec{n}} \in L^{2}\left(\mathbb{R}^{n}\right)$, to the sequence $\left(A^{\otimes n} \varphi_{\vec{n}}\right), n \in \mathrm{N}_{0}$, is called the second quantization of $A$. It is denoted by $\Gamma(A)$.

LEMMA 2.1 For bounded linear operators $A$ on $L^{2}(\mathbb{R}), \Gamma(A)$ is a continuous operator on $\mathcal{G}^{-1}$.

Proof. Given a bounded linear operator $A$ on $L^{2}(\mathbb{R})$, for every element $\Phi \in \mathcal{G}^{-1}$ (belonging to $G_{-q}^{-1}$, for some $q$ ), we have,

$$
\sum_{\vec{n}} 2^{-p n}\left|A^{\otimes n} \Phi_{\vec{n}}\right|_{n}^{2} \leq \sum_{\vec{n}} 2^{-p n}\|A\|^{2 n}\left|\Phi_{\vec{n}}\right|_{n}^{2},
$$

for every non negative integer number $p$. If $\|A\|^{2} \leq 2^{p-q}$ for some $p \in \mathrm{N}_{0}$ the above sum is majorized by $\|\Phi\|_{G_{-q}^{-1}}^{2}$ and the second quantization of $A$ is a bounded linear operator from $G_{-q}^{-1}$ into $G_{-p}^{-1}$. In particular, if $\|A\| \leq 1, \Gamma(A)$ is a bounded linear operator from $\mathcal{G}^{-1}$ into itself. The lemma is proved.

In particular, for $A=\Theta_{t}$, for some $t \in \mathbb{R}$, it follows that

$$
\left\|\Gamma\left(\Theta_{t}\right) \Phi\right\|_{G_{-q}^{-1}}^{2} \leq \sum_{\vec{n}} 2^{-q n}\left|\Theta_{t}^{\otimes n} \Phi_{\vec{n}}\right|_{n}^{2} \leq\|\Phi\|_{G_{-q}^{-1}}^{2},
$$

for every $q \in \mathrm{N}_{0}$. Hence $\Gamma\left(\Theta_{t}\right)$ is a bounded linear operator from the space of regular generalized functions $\mathcal{G}^{-1}$ into itself.

Remark 2. Consider the $\sigma$-algebra $\mathcal{F}_{t}$ generated by the random variables $\{B(s), s \leq t\}$. $\Gamma\left(\Theta_{t}\right) \Phi$ coincides with the conditional expectation for elements $\Phi$ from $\mathcal{G}^{-1}$ with respect to $\mathcal{F}_{t}$, as introduced in [8].

\section{THE GENERALIZED CLARK-OCONE FORMULA}

Now we are prepared to present the main result of this note. It generalizes the well known Clark-Ocone formula to regular generalized functions of white noise, i.e., to the space $\mathcal{G}^{-1}$.

Theorem 3.1 (Generalized Clark-Ocone Formula) Let $\Phi$ be a regular generalized function, $\Phi \in \mathcal{G}^{-1}$. Then it can be written as a generalized Itô integral

$$
\Phi=\mathbf{E}(\Phi)+I(m)
$$

with

$$
m_{i}(t)=\Gamma\left(\Theta_{t}\right) \partial_{t}^{i} \Phi
$$


Proof. We begin by noting that the integrand $m$ is non-anticipating. Let $\Phi$ be an arbitrary element from $\mathcal{G}^{-1}$, i.e., $\Phi \in G_{-q}^{-1}$ for some $q$, characterized by the sequence $\left(\Phi_{\bar{n}}\right), n \in \mathbb{N}_{0}$. Hence, for every test function $\psi \in \mathcal{G}^{1}$ with kernel functions given by $\left(\psi_{\vec{n}}\right), n \in \mathrm{N}_{0}$, we have

$$
\ll \Phi, \psi \gg=\mathbf{E}(\Phi) \mathbf{E}(\psi)+\sum_{\vec{n}, n \geq 1} \vec{n} !\left(\Phi_{\vec{n}}, \psi_{\vec{n}}\right)_{n},
$$

where, for each $n$-tuple $\vec{n}=\left(n_{1}, \ldots, n_{d}\right)$ such that $n \geq 1$,

$$
\left(\Phi_{\vec{n}}, \psi_{\vec{n}}\right)_{n}=\int_{\mathbb{R}^{n}} \mathrm{~d}^{n} s \Phi_{\vec{n}}\left(\ldots ; s_{1}^{i}, \ldots, s_{n_{i}}^{i} ; \ldots\right) \psi_{\vec{n}}\left(\ldots ; s_{1}^{i}, \ldots, s_{n_{i}}^{i} ; \ldots\right) .
$$

Taking each variable $s_{j}^{i}, j=1, \ldots, n_{i}, i=1, \ldots, d$, in term, in the range $s_{j}^{i} \geq$ $\sup _{i, j} s_{k}^{l}$, the above integral can be written as $(l, k) \neq(i, j)$

$\sum_{i=1}^{d} \sum_{j=1}^{n_{i}} \int_{\mathbb{R}} \mathrm{d} s_{j}^{i} \int_{-\infty}^{s_{j}^{i}} \mathrm{~d}^{n-1} s \Phi_{\bar{n}}\left(\ldots ; s_{1}^{i}, \ldots, s_{j}^{i}, \ldots, s_{n_{i}}^{i} ; \ldots\right) \psi_{\bar{n}}\left(\ldots ; s_{1}^{i}, \ldots, s_{j}^{i}, \ldots, s_{n_{i}}^{i} ; \ldots\right)$, which is equal to

$$
\sum_{i=1}^{d} n_{i} \int_{\mathbb{R}} \mathrm{d} \tau \int_{-\infty}^{\tau} d^{n-1} s \Phi_{\bar{n}}\left(\ldots ; \tau, s_{1}^{i}, \ldots, s_{n_{i}-1}^{i} ; \ldots\right) \psi_{\bar{n}}\left(\ldots ; \tau, s_{1}^{i}, \ldots, s_{n_{i}-1}^{i} ; \ldots\right),
$$

by the symmetry of $\Phi_{\vec{n}}$ and the kernel function $\psi_{\vec{n}}$ with respect to each $n_{i}$-tuple of variables $\left(s_{1}^{i}, \ldots, s_{n_{\mathrm{i}}}^{i}\right), i=1, \ldots, d$. This means,

$$
\begin{aligned}
& \ll \Phi, \psi \gg-\mathbf{E}(\Phi) \mathbf{E}(\psi)=\sum_{\vec{n}, n \geq 1} \vec{n} ! \sum_{i=1}^{d} n_{i} \int_{\mathbb{R}} \mathrm{d} \tau\left(\Theta_{\tau}^{\otimes(n-1)} \Phi_{\bar{n}}(\cdot, \tau, \cdot), \psi_{\vec{n}}(\cdot, \tau, \cdot)\right)_{n-1} \\
& =\sum_{i=1}^{d} \int_{\mathbb{R}} \mathrm{d} \tau \sum_{\vec{n}, n_{i} \geq 1} \vec{n} ! n_{i}\left(\Theta_{\tau}^{\otimes(n-1)} \Phi_{\vec{n}}(\cdot, \tau, \cdot), \psi_{\vec{n}}(\cdot, \tau, \cdot)\right)_{n-1} \\
& =\sum_{i=1}^{d} \int_{\mathbb{R}} \mathrm{d} \tau \sum_{\vec{n}, n_{i} \geq 1}\left(\overrightarrow{\left.n-\delta_{i}\right)}\right) !\left(\Theta_{\tau}^{\otimes(n-1)} n_{i} \Phi_{\vec{n}}(\cdot, \tau, \cdot), n_{i} \psi_{\vec{n}}(\cdot, \tau, \cdot)\right)_{n-1}
\end{aligned}
$$

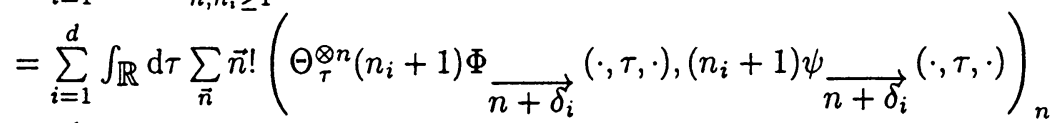

$$
\begin{aligned}
& =\sum_{i=1}^{d} \ll \Gamma(\Theta .) \partial^{i} \Phi, \partial^{i} \psi \gg \text {. }
\end{aligned}
$$

Therefore,

$$
\ll \Phi, \psi \gg=\mathbf{E}(\Phi) \mathbf{E}(\psi)+\sum_{i=1}^{d} \ll I_{i}\left(\Gamma(\Theta .) \partial^{i} \Phi\right), \psi \gg,
$$

for every $\psi$ belongs to $\mathcal{G}^{1}$, which implies the result. Theorem 3.1 is proved.

\section{S-TRANSFORM}

In this section, we find an expression to the $S$-transform of a regular generalized function $\Phi \in \mathcal{G}^{-1}$ which corresponds to the Clark-Ocone formula established above. 
THEOREM 4.1 Given a regular generalized function $\Phi$ from $\mathcal{G}^{-1}$ and $q \in \mathrm{N}_{0}$ such that $\Phi \in G_{-q}^{-1}$, its $S$-transform is equal to

$$
S \Phi(\vec{\eta})=\mathbf{E}(\Phi)+\sum_{i=1}^{d} \int_{\mathbb{R}} \mathrm{d} \tau \eta_{i}(\tau) \frac{\delta}{\delta \eta_{i}(\tau)} S(\Phi)\left(\Theta_{\tau} \vec{\eta}\right),
$$

for every $\vec{\eta}=\left(\eta_{1}, \ldots, \eta_{d}\right) \in U_{q}$.

Proof. Taking $\Phi \in \mathcal{G}^{-1}$ characterized by the sequence $\left(\Phi_{\vec{n}}\right), n \in \mathrm{N}_{0}$, such that $\Phi \in G_{-q}^{-1}$ for some $q \in \mathrm{N}_{0}$, for every test function $\vec{\eta}=\left(\eta_{1}, \ldots, \eta_{d}\right) \in S_{d, c}(\mathbb{R})$ with $2^{q}|\vec{\eta}|_{L_{d}^{2}(\mathbb{R})}^{2}<1$ we have

$$
S \Phi(\vec{\eta})=\mathrm{E}(\Phi)+\sum_{i=1}^{d} \ll I_{i}\left(\Gamma(\Theta .) \partial^{i} \Phi\right),: \exp \langle\cdot, \vec{\eta}>: \gg .
$$

Here,

$$
\sum_{i=1}^{d} \ll I_{i}\left(\Gamma(\Theta .) \partial^{i} \Phi\right),: \exp \langle\cdot, \vec{\eta}\rangle: \gg=\sum_{\vec{n}, n \geq 1}\left(\Phi_{\vec{n}}, \vec{\eta}^{\otimes \vec{n}}\right)_{n} .
$$

Using the symmetry of $\Phi_{\vec{n}}$ in each $n_{i}$-tuple of variables $\left(s_{1}^{i}, \ldots, s_{n_{i}}^{i}\right), i=1, \ldots, d$, it follows that

$$
\begin{aligned}
\left(\Phi_{\vec{n}}, \vec{\eta}^{\otimes \vec{n}}\right)_{n}= & \int_{\mathbb{R}^{n}} \mathrm{~d}^{n} s \Phi_{\vec{n}}\left(\ldots ; s_{1}^{i}, \ldots, s_{n_{i}}^{i} ; \ldots\right) \prod_{k=1}^{d} \eta_{k}\left(s_{1}^{k}\right) \cdots \eta_{k}\left(s_{n_{k}}^{k}\right) \\
= & \sum_{i=1}^{d} \sum_{j=1}^{n_{i}} \int_{\mathbb{R}} \mathrm{d} s_{j}^{i} \int_{-\infty}^{s_{j}^{i}} \mathrm{~d}^{n-1} s \Phi_{\vec{n}}\left(\ldots ; s_{1}^{i}, \ldots, s_{n_{i}}^{i} ; \ldots\right) \prod_{k=1}^{d} \eta_{k}\left(s_{1}^{k}\right) \cdots \eta_{k}\left(s_{n_{k}}^{k}\right) \\
= & \sum_{i=1}^{d} n_{i} \int_{\mathbb{R}} \mathrm{d} \tau \int_{-\infty}^{\tau} \mathrm{d}^{n-1} s \Phi_{\vec{n}}\left(\ldots ; \tau, s_{1}^{i} \ldots, s_{n_{i}-1}^{i} ; \ldots\right) . \\
& \times \eta_{i}(\tau) \eta_{i}\left(s_{1}^{i}\right) \cdots \eta_{i}\left(s_{n_{i}-1}^{i}\right) \prod_{k=1, k \neq i}^{d} \eta_{k}\left(s_{1}^{k}\right) \cdots \eta_{k}\left(s_{n_{k}}^{k}\right) \\
= & \sum_{i=1}^{d} \int_{\mathbb{R}} \mathrm{d} \tau \eta_{i}(\tau)\left(n_{i} \int_{-\infty}^{\tau} \mathrm{d}^{n-1} s \Phi_{\vec{n}}(\cdot, \tau, \cdot) \overrightarrow{\eta^{\otimes n-\delta_{i}}}\right) .
\end{aligned}
$$

Thus

$$
\sum_{\vec{n}, n \geq 1}\left(\Phi_{\vec{n}}, \vec{\eta}^{\otimes \vec{n}}\right)_{n}=\sum_{i=1}^{d} \int_{\mathbb{R}} \mathrm{d} \tau \eta_{i}(\tau) \mu_{i}(\tau)
$$

where

$$
\mu_{i}(\tau) \equiv \sum_{\vec{n}, n_{i} \geq 1} n_{i} \int_{-\infty}^{\tau} \mathrm{d}^{n-1} s \Phi_{\vec{n}}(\cdot, \tau, \cdot) \vec{\eta}^{\otimes n-\delta_{i}}, \tau \in \mathbb{R} .
$$

But, for each $\tau$,

$$
\begin{aligned}
\mu_{i}(\tau) & =\sum_{\vec{n}, n_{i} \geq 1}\left(n_{i} \Theta_{\tau}^{\otimes n-1} \Phi_{\vec{n}}(\cdot, \tau, \cdot), \vec{\eta}^{\otimes} \overrightarrow{n-\delta_{i}}\right)_{n-1} \\
& =S\left(\Gamma\left(\Theta_{\tau}\right) \partial_{\tau}^{i} \Phi\right)(\vec{\eta}) .
\end{aligned}
$$


Hence, (6) can be written as

$$
S \Phi(\vec{\eta})=\mathbf{E}(\Phi)+\sum_{i=1}^{d} \int_{\mathbb{R}} \mathrm{d} \tau \eta_{i}(\tau) S\left(\Gamma\left(\Theta_{\tau}\right) \partial_{\tau}^{i} \Phi\right)(\vec{\eta}) .
$$

Observing that, for each $\tau$,

$$
\begin{aligned}
S\left(\Gamma\left(\Theta_{\tau}\right) \partial_{\tau}^{i} \Phi\right)(\vec{\eta}) & =\sum_{\vec{n}}\left(n_{i}+1\right) \int_{\mathbb{R}^{n}} \mathrm{~d}^{n} s \Phi{\overrightarrow{n+\delta_{i}}}_{\vec{n}^{\prime}}(\cdot, \tau, \cdot)\left(\Theta_{\tau} \vec{\eta}\right)^{\otimes \vec{n}} \\
& =\frac{\delta}{\delta \eta_{i}(\tau)} S(\Phi)\left(\Theta_{\tau} \vec{\eta}\right),
\end{aligned}
$$

there follows the required equality

$$
S \Phi(\vec{\eta})=\mathrm{E}(\Phi)+\sum_{i=1}^{d} \int_{\mathbb{R}} \mathrm{d} \tau \eta_{i}(\tau) \frac{\delta}{\delta \eta_{i}(\tau)} S(\Phi)\left(\Theta_{\tau} \vec{\eta}\right) .
$$

Theorem 4.1 is proved.

\section{AN EXAMPLE}

As an application of the above let us consider $\Phi$ equal to the Donsker delta function which we may consider defined as a Fourier (Bochner) integral [10]

$$
\delta(B(t)-a)=\frac{1}{2 \pi} \int_{\mathbb{R}} \mathrm{d} \lambda \mathrm{e}^{\mathrm{i} \lambda(B(t)-a)},
$$

with S-transform

$$
(S \delta(B(t)-a))(f)=\frac{1}{\sqrt{2 \pi t}} \exp \left(-\frac{\left(\int_{0}^{t} f(s) \mathrm{d} s-a\right)^{2}}{2 t}\right), f \in S_{c}(\mathbb{R}) .
$$

It is well known that $\delta(B(t)-a)$ is in $\mathcal{G}^{-1}$. From Theorems 3.1 and 4.1 it follows that

$$
\delta(B(t)-a)=\mathbf{E}(\delta(B(t)-a))+\int \mathrm{d} B(\tau) m(\tau)
$$

with

$$
S m(\tau)(f)=\frac{\delta}{\delta f(\tau)} S(\Phi)\left(\Theta_{\tau} f\right) .
$$

The functional derivative of (7) is calculated straightforwardly using (5)

$$
\left(\frac{\delta}{\delta f(\tau)} S(\Phi)\right)(f)=-\frac{1_{[0, t]}(\tau)}{\sqrt{2 \pi t^{3}}}\left(\int_{0}^{t} f(s) \mathrm{d} s-a\right) \exp \left(-\frac{\left(\int_{0}^{t} f(s) \mathrm{d} s-a\right)^{2}}{2 t}\right)
$$

(here, $1_{[0, t]}$ denotes the indicator function of the interval $[0, t]$ ), so that, projecting the $f$ with $\Theta_{\tau}$, we obtain

$$
(S m(\tau))(f)=-\frac{1_{[0, t]}(\tau)}{\sqrt{2 \pi t^{3}}}\left(\int_{0}^{\tau} f(s) \mathrm{d} s-a\right) \exp \left(-\frac{\left(\int_{0}^{\tau} f(s) \mathrm{d} s-a\right)^{2}}{2 t}\right) .
$$


Note that the rhs depends only on

$$
\lambda \equiv \int f(s) e(s) \mathrm{d} s
$$

where $e=\frac{1}{\sqrt{\tau}} 1_{[0, \tau]}$ is a unit vector in $L^{2}(\mathbb{R})$. Consequently, $m$ depends only on the normal random variable

$$
x=\left\langle\omega, e>=\frac{1}{\sqrt{\tau}} B(\tau)\right.
$$

and

$$
m(\tau)=h\left(\frac{1}{\sqrt{\tau}} B(\tau)\right)
$$

with $S$-transform

$$
\int \frac{\mathrm{d} x}{\sqrt{2 \pi}} e^{-\frac{1}{2} x^{2}} h(x) \mathrm{e}^{\lambda x} \mathrm{e}^{-\frac{1}{2} \lambda^{2}}=-\frac{1_{[0, t]}(\tau)}{\sqrt{2 \pi t^{3}}}(\sqrt{\tau} \lambda-a) \exp \left(-\frac{(\sqrt{\tau} \lambda-a)^{2}}{2 t}\right) .
$$

To obtain $m$ itself we must thus calculate the inverse Laplace transform of

$$
q(\lambda)=-\frac{1_{[0, t]}(\tau)}{\sqrt{t^{3}}}(\sqrt{\tau} \lambda-a) \mathrm{e}^{-\frac{(\sqrt{\tau} \lambda-a)^{2}}{2 t}} \mathrm{e}^{\frac{1}{2} \lambda^{2}}
$$

which gives

$$
h(x)=-\frac{1_{[0, t]}(\tau)}{\sqrt{t^{3}}}\left(\frac{t}{t-\tau}\right)^{3 / 2}(\sqrt{\tau} x-a) \exp \left(-\frac{(\sqrt{\tau} x-a)^{2}}{2(t-\tau)}\right) .
$$

Substituting

$$
x=\frac{1}{\sqrt{\tau}} B(\tau)
$$

we finally obtain

$$
m(\tau)=-\frac{1_{[0, t]}(\tau)}{\sqrt{(t-\tau)^{3}}}(B(\tau)-a) \exp \left(-\frac{(B(\tau)-a)^{2}}{2(t-\tau)}\right) .
$$

One notes that $m(\tau)$ is an adapted random variable in $\left(L^{2}\right)$ as long as $\tau<t$, and it permits conventional Itô integration. It is thus not hard to show that, as a limit in $\mathcal{G}^{-1}$,

$$
\delta(B(t)-a)=\frac{1}{\sqrt{2 \pi t}} \mathrm{e}^{-\frac{a^{2}}{2 t}}+\lim _{\varepsilon \rightarrow+0} \int_{0}^{t-\varepsilon} \mathrm{d} B(\tau) m(\tau) .
$$

\section{Acknowledgments}

This work has had partial support from PRAXIS XXI and FEDER. M. J. O. is grateful for an encouraging discussion with Prof. B. Øksendal, and for hospitality at CCM; L. S. would like to express his gratitude for the generous hospitality of the Grupo de Física Matemática da Universidade de Lisboa, under the auspices of a "Marie Curie" fellowship (ERBFMBICT 971949). Prof. Yu. Kondratiev improved the manuscript by many helpful comments. 


\section{REFERENCES}

1. K. Aase, B. Øksendal, J. Ubøe. White noise generalizations of the Clark-Ocone theorem with application to mathematical finance. MaPhySto Research Report $n^{o}$ 30, University of Aarhus (1998).

2. K. Aase, B. Øksendal, J. Ubøe. Using the Donsker delta function to compute hedging strategies. Preprint University of Oslo $n^{\circ} 6$ (1998).

3. F. E. Benth. An Addendum to 'An Introduction to Malliavin Calculus with Applications to Economics'. MaPhySto Miscellanea n ${ }^{\circ} 1$, University of Aarhus (1998).

4. F. E. Benth, J. Potthoff. On the martingale property for generalized stochastic processes, Stochastics Stochastics Rep. 58 (3-4), 349-367 (1996).

5. J. M. C. Clark. The representation of functionals of Brownian motion by stochastic integrals, Ann. Math. Stat. 41 1282-1295 (1970) and 42, 1778 (1971).

6. Th. Deck, J. Potthoff, G. Vage. A review of white noise analysis from a probabilistic standpoint. Acta Appl. Math. 48, 91-112 (1997).

7. M. de Faria, C. Drumond, L. Streit. The renormalization of self intersection local times. I: The chaos expansion. Preprint IFM 1/98, Grupo de Física Matemática da Universidade de Lisboa (1998).

8. M. Grothaus, Yu. G. Kondratiev, L. Streit. Regular generalized functions in Gaussian analysis. Preprint 23/97, Universidade da Madeira, 1997. To appear in Infinite Dim. Anal. Quantum Prob. 2 (1)(1999).

9. M. Grothaus, Yu. G. Kondratiev, G. F. Us. Wick calculus for regular generalized stochastic functionals, Random Oper. Stochastic Equations 7, 3, 263-290, (1999).

10. T. Hida, H. H. Kuo, J. Potthoff, L. Streit. White Noise. An Infinite Dimensional Calculus. Kluwer, Dordrecht (1993).

11. Yu. G. Kondratiev, P. Leukert, L. Streit. Wick calculus in Gaussian analysis. Acta Appl. Math. 44, 269-294 (1996).

12. H. H. Kuo. White Noise Distribution Theory. CRC Press, Boca Raton (1996).

13. N. Obata. White Noise Calculus and Fock Space. Lecture Notes in Math. 1577, Springer-Verlag (1994).

14. D. Ocone. Malliavin's calculus and stochastic integral representations of functionals of diffusion processes, Stochastics 12, 161-185 (1984).

15. B. Øksendal. An introduction to Malliavin calculus with applications to economics. Working Paper $n^{\circ} 3 / 96$, Norwegian School of Economics and Business Administration (1996).

16. J. Potthoff, M. Timpel. On a dual pair of spaces of smooth and generalized random variables, Potential Analysis 4, 637-654 (1995).

17. A. S. Üstünel. Representation of the distributions on Wiener space and stochastic calculus of variations, JFA 70, 126-139 (1987). 